\title{
L'intercorporéité au-delà du " je peux » : Husserl, Merleau-Ponty et Levinas
}

Paula Lorelle

\section{OpenEdition}

\section{Journals}

Édition électronique

URL : http://journals.openedition.org/alter/392

DOI : $10.4000 /$ alter.392

ISSN : 2558-7927

Éditeur :

Association ALTER, Archives Husserl (CNRS-UMR 8547)

\section{Édition imprimée}

Date de publication : 1 novembre 2015

Pagination : 245-260

ISBN : 978-2-9550449-1-9

ISSN : 1249-8947

\section{Référence électronique}

Paula Lorelle, «L'intercorporéité au-delà du « je peux » : Husserl, Merleau-Ponty et Levinas », Alter [En ligne], 23 | 2015, mis en ligne le 01 décembre 2017, consulté le 03 mai 2019. URL : http:// journals.openedition.org/alter/392; DOI : 10.4000/alter.392 


\section{L'INTERCORPORÉITÉ AU-DELÀ DU « JE PEUX » : HUSSERL, MERLEAU-PONTY ET LEVINAS}

Paula Lorelle

Cet article entend faire se confronter deux fameux motifs de la tradition phénoménologique inaugurés par Husserl et développés par Merleau-Ponty: (1) le motif du «je peux» comme mode distinctif du corps propre (Leib); (2) et le motif de l'intercorporéité comme mode originaire de l'intersubjectivité. L'intercorporéité implique-telle le "je peux» comme unique mode de la corporéité ? "Tout ce que je vois par principe est à ma portée, écrit Merleau-Ponty, du moins à la portée de mon regard, relevé sur la carte du "je peux" ${ }^{1}$. Mais qu'en est-il donc du corps d'autrui ?

L'intercorporéité désigne la dimension originairement esthésiologique de l'intersubjectivité. Autrui serait d'abord un autre corps. «Ce que je perçois d'abord, comme l'écrit encore Merleau-Ponty, c'est une autre "sensibilité" (Empfindbarkeit), et, à partir de là seulement, un autre homme et une autre pensée $»^{2}$. Ce concept d'intercorporéité résulte semble-t-il d'une radicalisation de l'analyse husserlienne de $l^{\prime}$ « apprésentation ». Dès la cinquième Méditation cartésienne, l'Einfühlung comme relation aux vécus psychiques d'autrui, présuppose l'apprésentation ou l'aperception par analogie. Les « contenus déterminés de la sphère psychique supérieure [...] nous sont suggérés, indiqués, eux aussi, écrit Husserl, par le corps et par le comportement de l'organisme dans le monde extérieur $»^{3}$. La compréhension d'autrui comme alter ego, présuppose l'apprésentation analogique de son corps

\footnotetext{
${ }^{1}$ M. Merleau-Ponty, CEuvres, L'œil et l'esprit, Paris, Gallimard, 2010, p. 1594.

${ }^{2}$ M. Merleau-Ponty, "Le philosophe et son ombre », in CEuvres, Paris, Gallimard, 2010, p. 1277.

${ }^{3}$ E. Husserl, Husserliana I : Cartesianische Meditationen, S. Strasser (éd.), La Haye, Martinus Nijhoff, 1950, p. 149 ; trad. G. Peiffer et E. Levinas, Méditations cartésiennes, Paris, Vrin, 1992, p. 194-195 (Cité Нua I/MC).
} 
comme autre corps organique (Leib). Or, puisque je n'ai pas accès aux vécus corporels $d$ 'autrui comme $j$ 'ai accès aux miens, "seule une ressemblance reliant dans ma sphère primordiale cet autre corps avec le mien, peut fournir le fondement et le motif de concevoir "par analogie" ce corps comme un autre organisme $»^{4}$.

La radicalisation merleau-pontienne de $l^{\prime}$ « apprésentation » abolit la dimension d'extranéité originaire du corps d'autrui que présuppose encore le concept $d^{\prime}$ « analogie ». Si l'aperception par analogie implique bien ce que Husserl nomme un «accouplement (Paarung) au sens d'une " unité de ressemblance » donnée intuitivement, cette unité reste une unité de ressemblance justement, qui présuppose l'altérité originaire $\mathrm{du}$ corps d'autrui. La nécessité même d'une perception médiate d'autrui par analogie présuppose la séparation première d'une sphère $d$ 'appartenance et de ce qui lui est étranger - la priorité de l'expérience de soi sur l'expérience d'autrui. La perception d'autrui est médiatisée par ma perception interne et l'inter-corporéité désigne ici la simple relation externe de mon corps à un corps étranger. Si Husserl admet bien la priorité d'une perception corporelle d'autrui sur la perception de sa pensée, il continue dans les Méditations cartésiennes, à penser le corps sur le modèle de l'ego - comme le fait d'une sphère d'appartenance primordiale.

Radicaliser cette conception «esthésiologique » de l'intersubjectivité, c'est libérer le corps de cette objectivation, et faire retour à l'unité originaire de la comprésence qu'il forme avec le corps d'autrui. En ce sens, l'inter-corporéité ne désigne plus la relation externe de deux corps étrangers mais bien la relation interne de deux corps qui partagent une même chair. C'est au second tome des Idées directrices que Merleau-Ponty dans "Le philosophe et son ombre », voit déjà s'esquisser cette conception de l'intercorporéité. Husserl cherche à faire retour d'une attitude théorique qui a affaire à de "pures choses " (blosze Sachen), à l'attitude pré-théorique qu'elle implique et au sein de laquelle l'opposition objectivante du même et de l'autre est déjouée ${ }^{5}$. Ce monde de pures choses au sein duquel je m'oppose à autrui, présuppose un "monde commun » au sein duquel je suis toujours déjà avec lui. Un monde d'intercorporéité primordiale, tissé d'une chair qui comprend nos corps et les excède. La thèse d'une priorité de l'intercorporéité sur l'intropathie prend donc une toute autre tournure, dès lors que la corporéité n'est plus pensée comme

\footnotetext{
${ }^{4}$ Ibid., p. 140 ; ibid., p. 180.

${ }^{5}$ Voir à ce propos l'introduction d'E. Escoubas au second tome des Ideen. E. Husserl, Recherches phénoménologiques pour la constitution, trad. E. Escoubas, Paris, PUF, 1982, p. 14.
} 
une sphère égoïque d'appartenance primordiale, mais comme l'appartenance primordiale à un monde commun. L'intersubjectivité se joue en deçà de toute pensée, à un niveau premier qui comprend toujours déjà le pluralisme de la société. Alors que la conscience théorique se dessine à l'exclusion de l'altérité, le sensible, lui, est essentiellement ouvert - il « ne prétend pas au monopole de l'être, et n'institue pas la lutte à mort des consciences $»^{6}$. Ainsi la corporéité dont il $\mathrm{s}^{\prime}$ agit de penser la dimension « relationnelle » ne désigne plus ici mon propre corps organique, mais la « corporéité en générale ${ }^{7}$, comme la chair originaire toujours déjà tissée d'une pluralité de corps animés. L'intercorporéité peut donc se passer d'analogie :

Il faut bien voir qu'il n'y a là ni comparaison, ni analogie, ni projection, ou "introjection». Si, en serrant la main de l'autre homme, j'ai l'évidence de son être-là, c'est qu'elle se substitue à ma main gauche, que mon corps annexe le corps d'autrui dans cette sorte de "réflexion" dont il est paradoxalement le siège. Mes deux mains sont "comprésentes" ou "coexistent » parce qu'elles sont les mains d'un seul corps: autrui apparaît par extension de cette comprésence, lui et moi sommes comme les organes d'une seule intercorporéité ${ }^{8}$.

Organe d'une seule et même chair, « autrui n'est pas impossible » affirme Merleau-Ponty ${ }^{9}$. Mais en quel sens de « possibilité » et d' «impossibilité », autrui n'est-il pas «impossible » à proprement parler? La thèse d'une intercorporéité primordiale implique, comme cette étude entend le montrer, le motif du « je peux». Le sens de la " possibilité » que désigne le « je peux » doit être ici entendu dans toute son équivocité : de la "possibilité logique » de se représenter à la «possibilité effective» de « rendre intuitive cette possibilité »; de la «possibilité logico-doxique » de l'existence d'un objet, à la "possibilité pratique » d'un " pouvoir d'agir » sur lui ${ }^{10}$; ou encore, de la " possibilité » d'un corps-sujet, à la « compossibilité » dont serait d'emblée tissé le monde de l'intersubjectivité. De même est-ce en plusieurs sens qu'il sera possible de parler des "possibles» du corps comme de

\footnotetext{
${ }^{6}$ M. Merleau-Ponty, « Le philosophe et son ombre », in CEuvres, op. cit., p. 1278.

${ }^{7}$ Ibid., p. 1283.

${ }^{8}$ Ibid., p. 1276-1277.

${ }^{9}$ Ibid., p. 1278-1279.

10 À propos de ces deux dernières distinctions, voir E. Husserl, Husserliana IV : Ideen zur einer reinen Phänomenologie und phänomenologischen Philosophie, Zweites Buch, M. Biemel (éd.), La Haye, Martinus Nijhoff, 1952, p. 261-262 ; trad. E. Escoubas, Recherches phénoménologiques pour la consti-

tution, Paris, PUF, 1982, p. 354-355 (Cité Hua IV ; RPC). Voir également à ce propos, L. Perreau, Le monde social selon Husserl, New York/Londres, Springer, 2013, p. 241.
} 
« pouvoirs » à proprement parler - au sens « logique »d'un pouvoir de représentation - intuitive ou non - et au sens «pratique » d'un pouvoir d'action. C'est en ces différents sens qu'il s'agira donc de se demander si le "je peux » épuise le phénomène de l'intercorporéité. Est-ce là la seule dimension de la corporéité impliquée par la possibilité d'une relation charnelle à l'altérité ? Tout se passe à vrai dire comme si le concept merleau-pontien $\mathrm{d}^{\prime}$ « intercorporéité » tendait à s'enferrer dans une alternative trop stricte avec la voie égologique dont il entend résoudre les apories. À la séparation des consciences, s'opposerait l'union des corps; à l'impossibilité de la conscience d'autrui, s'opposerait la chair sensible comme "compossibilité ». Mais n'y a-t-il pas de la séparation à même les corps? De l'impossibilité à même l'intercorporéité ? Et après Husserl, qui mieux que Levinas aura pensé cette déchirure de l'altérité à même la chair interpersonnelle?

Il s'agira donc dans un premier temps de distinguer les différentes figures de la "possibilité corporelle » impliquées par le phénomène de l'intercorporéité. Puis nous tâcherons, au moyen d'une mise en question des principes respectifs sur lesquels reposent ces différentes conceptions, de faire s'esquisser le «je ne peux pas» qui, chez Husserl déjà, est tout aussi constitutif de l'intercorporéité. Ce, pour conclure sur la manière dont l'intercorporéité peut être pensée avec Levinas au-delà de tout pouvoir comme de tout système de compossibilité.

\section{Le « je peux » et « l'autre existe »}

Deux différentes conceptions de la «possibilité corporelle » peuvent être interrogées du point de vue des rôles respectifs qu'elles jouent dans l'intercorporéité : le pouvoir de perception et d'action du corps propre ou du corps-sujet; et la «compossibilité » qui désigne l'essentielle coexistence de nos possibilités corporelles et de celles d'autrui.

\section{$a-L e$ « je peux »du corps-propre}

C'est en deux sens que le corps-propre est pensé par Husserl comme l'organe d'un pouvoir. (1) Au sens tout d'abord où il est le lieu premier et immédiat du pouvoir de l'ego - ce sur quoi la conscience peut. (2) Et au sens ensuite où il est à son tour ce qui peut sur 
les choses - ce qui peut les percevoir et les transformer. C'est parce que le corps est « pu » par le sujet, qu'il «peut » sur le monde. Si mon corps propre se donne comme corps organique (Leib), se distinguant d'un simple corps matériel (Körper), c'est d'ailleurs parce que j'en dispose et peux le régir, comme l'affirme Husserl au $\S 44$ des Méditations cartésiennes. Et comme il l'affirme tout aussi explicitement dans les Ideen II, le corps propre est « l'organe du vouloir (Willensorgan) [...] le seul et unique objet qui peut être mis en mouvement de manière spontanée et immédiate par le vouloir de l'ego pur qui est le mien $»^{11}$. La spontanéité et l'immédiateté caractérisent le pouvoir qu'a l'ego sur son corps. Le sujet "a la "faculté" ("je peux") de mouvoir librement ce corps ${ }^{12}$. Et, ainsi «pu», le corps peut pouvoir à son tour. "J'ai pouvoir sur mon corps, et ce n'est que du fait que j'ai pouvoir sur mon corps que j'ai pouvoir sur le monde physique ${ }^{13}$.

Ce pouvoir sur le monde est d'abord celui de le percevoir, et le corps est d'abord, selon une expression de Husserl, «moyen » ou "organe de perception ${ }^{14}$. Ce, du fait premier de son appartenance aux choses, ou de sa localisation, et du fait second de son mouvement. Comme l'écrit Merleau-Ponty à ce propos, " entre les mouvements de mon corps et les "propriétés" de la chose qu'ils révèlent, le mouvement est celui du "je peux" »" C'est en se mouvant à même les choses, que le corps peut en révéler les propriétés. Et ce pouvoir de perception est toujours corrélatif d'un pouvoir d'action, comme l'écrit Husserl au $\S 44$ des Méditations cartésiennes:

Je perçois avec les mains, (c'est par les mains que j'ai - et que je puis toujours avoir - des perceptions cinesthésiques tactiles), avec les yeux (c'est par les yeux que je vois), etc. ; et ces phénomènes cinesthésiques des organes forment un flux de modes d'action et relèvent de mon "je peux ». Je peux ensuite, en mettant en jeu ces phénomènes cinesthésiques, heurter, pousser, etc., et ainsi agir par mon corps, immédiatement d'abord, et à l'aide d'autres choses (médiatement) ensuite ${ }^{16}$.

Dès lors que le pouvoir immédiat du corps sur le monde, luimême déterminé par le pouvoir immédiat de l'esprit sur le corps, n'est plus seulement le pouvoir théorique de la perception, mais bien celui pratique de l'action, l'on devine toute l'importance de sa possi-

\footnotetext{
${ }^{11}$ Hua IV, p. 151-152 ; RPC, p. 215.

${ }^{12}$ Ibid., p. 152 ; p. 216.

${ }^{13}$ Ibid., p. 261 ; p. 354.

${ }^{14}$ Ibid., p. 56 ; p. 92.

${ }^{15}$ M. Merleau-Ponty, « Le philosophe et son ombre », in CEuvres, op. cit., p. 1274.

${ }^{16}$ Hua I, p. 128 ; MC, p. 159.
} 
ble transposition au problème de l'intercorporéité. Non seulement puis-je percevoir le corps d'autrui, mais je peux, qui plus est, agir sur lui.

Si cette seconde possibilité n'est pas, comme nous y reviendrons, suffisamment interrogée par Husserl ni par Merleau-Ponty, la thématique du corps comme «je peux » est bien liée pourtant à la possibilité de l'empathie ou à la perception médiate d'autrui par analogie. L'aperception par analogie n'est pas un « raisonnement », affirme Husserl, mais une "possibilité corporelle». Cette faculté corporelle relève d'une transposition aperceptive de mon corps au corps d'autrui et comprend deux opérations que sont: (1) l'accouplement (Paarung) passif - ou la manière dont mon corps se transpose passivement en autrui ${ }^{17}$; (2) la transposition imaginaire de l'ici (hic) au làbas (illic) - ou la possibilité qu'a mon corps de se " mettre à la place » $d^{\prime}$ 'autrui ${ }^{18}$. (1) L'accouplement est une forme de la synthèse passive dans laquelle mon corps et le corps d'autrui sont « intuitivement donnés dans l'unité d'une conscience » comme fondant phénoménologiquement une « unité de ressemblance » ${ }^{19}$. Ainsi le sens qu'a pour moi mon propre corps peut-il être transposé à l'ensemble de cette perception accouplante - et ipso facto, au corps d'autrui. (2) À cette première expérience de ressemblance, s'ensuit la possibilité d'une transposition spatiale imaginaire dans les phénomènes perceptifs d'autrui. Une fois le corps d'autrui passivement perçu comme autre corps organique qui perçoit lui aussi, il est possible de se transposer en lui, comme occupant cette autre position perceptive dans l'espace qui n'est pas celle de $\mathrm{l}^{\prime}$ « ici » (hic) mais celle du « là-bas » (illic). La possibilité imaginaire d'une telle transposition est fondée sur la possibilité effective de se déplacer dans l'espace. C'est parce que je peux me déplacer, que je peux appréhender les phénomènes perceptifs de l'autre, comme ceux que j'aurais moi-même si j'étais là-bas (illic) ${ }^{20}$.

Et, de la possibilité d'une transposition imaginaire dans les sensualia d'autrui, résulte la pénétration de ses propres possibilités - de

\footnotetext{
${ }^{17}$ Le « je peux » désigne donc d'abord une expérience dite «de pure passivité » - mais d'une passivité qui n'est pas à confondre néanmoins avec le "pâtir» qui, comme nous le verrons, relève d'une véritable inversion du «je peux». Le fait pour l'aperception accouplante d'être donnée dans une "pure passivité », est explicité dans ce texte comme le fait de pouvoir ou non être « remarquée» (ibid., p. $142 ;$ p. 183).

${ }^{18}$ Sur la distinction de ces deux premières étapes de l'empathie, voir notamment, N. Depraz, "The Husserlian Theory of Intersubjectivity as Alterology», Journal of Consciousness Studies, vol. 8, no 5-7, 2001, p. 172-173.

${ }^{19}$ Hua I, p. 142 ; p. 183.

${ }^{20}$ Ibid., p. 146 ; p. 190.
} 
son hic comme du champ de ses pouvoirs perceptifs localisés ${ }^{21}$. Percevoir l'autre comme alter ego revient donc en dernière instance, à percevoir ses propres possibilités, indices d'un monde intersubjectif de « compossibilité » où autrui «peut » lui aussi. Comme l'écrit Husserl au $\S 56$ des Méditations cartésiennes : «Et, en avançant ainsi, je reconnais que chaque monade qui possède la valeur d'une possibilité concrète détermine d'avance un univers compossible $»^{22}$.

\section{$b-$ La compossibilité}

La compossibilité est un concept d'origine leibnizienne et désigne une possibilité qui, plus restreinte que celle de la logique, dépend d'autres possibilités. Il n'est donc pas étonnant que Husserl emploie ce concept dans un contexte monadologique où il est question de la constitution d'une communauté intermonadique. Seulement, pour parler d' «intersubjectivité monadique », Husserl refuse l'idée d'une monade sans fenêtres dont la «compossibilité » ne serait permise qu'au moyen d'une harmonie préétablie: «Une monade a donc des fenêtres par lesquelles elle reçoit les influences étrangères. Ce sont les fenêtres de l'intropathie $»^{23}$. La compossibilité relève moins ici d'une harmonie préétablie entre des monades hermétiquement closes, que de leur commune possibilité corporelle de se comprendre l'une l'autre et de se comprendre comme possibilité commune de perception, d'action, voire de constitution.

La radicalisation merleau-pontienne $\mathrm{du}$ concept $\mathrm{d}^{\prime}$ «intercorporéité », implique la radicalisation corrélative du concept de «compossibilité ». De même que l'intercorporéité n'est pas la relation externe de deux corps séparés, la « compossibilité » ne résulte pas simplement de la rencontre d'une possibilité qui, en l'autre, serait semblable à la mienne. La possibilité est d'abord « compossibilité ». Contre la thèse des Méditations cartésiennes, l'intersubjectivité est moins précédée d'un solus ipse, que d'une "généralité primordiale où nous sommes confondus ${ }^{24}$; elle est moins précédée d'un corps et de ses possibilités isolées

\footnotetext{
${ }^{21}$ Voir Hua IV, p. 168 ; RPC, p. 370 : «Je me mets à la place de l'autre sujet: je saisis par l'intropathie ce qui le motive et quelle en est l'intensité, la force. J'apprends à comprendre du dedans comment il se comporte et se comporterait, étant donné que tels et tels motifs le déterminent avec telle force, et donc à comprendre ce dont il est capable ou non ».

${ }^{22}$ Hua I, p. 168 ; MC, p. 227.

${ }^{23}$ E. Husserl, Husserliana XIV, Zür Phänomenologie der Intersubjectivität, Zweiter Teil : 1921-1928, I. Kern (éd.), La Haye, Martinus Nijhoff, 1973, p. 295.

${ }^{24}$ M. Merleau-Ponty, « Le philosophe et son ombre », in CEuvres, op. cit., p. 1283.
} 
que d'un seul et même monde de compossibilité. Un monde au sein duquel le « je peux » et «l'autre existe » coexistent déjà :

Si, «à partir » du corps propre, je peux comprendre le corps et l'existence
d'autrui, si la "comprésence » de ma "conscience» et de mon "corps » se
prolonge dans la comprésence d'autrui et de moi, c'est que le «je peux » et
que «l'autre existe», appartiennent d'ores et déjà au même monde, que le
corps propre est prémonition d'autrui ${ }^{25}$.

Ce n'est donc pas parce que « je peux » que « l'autre existe », mais parce que le monde est d'abord tissé de cette compossibilité. Se référant implicitement à Sein und Zeit, Merleau-Ponty affirme en effet $\mathrm{qu}^{\prime}$ « il faut donc concevoir [...] un On primordial qui a son authenticité ${ }^{26}$. La compossibilité en vient donc de nouveau à désigner une forme d'harmonie - quoiqu'une harmonie essentiellement ouverte, qui comprend de l'écart et qui, en un sens spécifiquement phénoménologique, se déploie à même la chair interpersonnelle du sensible. C'est selon Merleau-Ponty, ce que Husserl nous aurait dit de plus profond dans ses Recherches phénoménologiques pour la constitution. Lecture qui rejoint également celle de Patočka dans ses cours sur Husserl : «Le corps et les choses, écrit-il, forment ensemble un système ${ }^{27}$. C'est parce qu'il est intégré à la "complexion du monde matériel ", que le corps peut agir sur lui ${ }^{28}$. Complexion essentiellement ouverte du monde dont Merleau-Ponty dit qu'elle comprend inversement une pluralité d'ek-stases ou d'ouvertures à lui ${ }^{29}$. Ainsi la possibilité en jeu dans l'intercorporéité désigne-t-elle moins la possibilité de «vivre le corps d'autrui »- la possibilité de vivre ses vécus et ses pensées - que la possibilité de vivre-avec-autrui, dans une coouverture au monde. Avant de pouvoir percevoir autrui dans le monde, je peux percevoir le monde avec lui.

L'on comprend donc pourquoi la possibilité d'agir, non plus « avec » mais «sur » autrui, n'est pas non plus abordée par MerleauPonty. La «compossibilité » permet moins encore de rendre compte de $\mathrm{l}^{\prime}$ « impossibilité » qui se déploie à même le phénomène sensible de l'intercorporéité.

\footnotetext{
${ }^{25}$ Ibid., p. 1284.

${ }^{26}$ Idem.

${ }^{27} \mathrm{~J}$. Patočka, Introduction à la phénoménologie de Husserl, trad. E. Abrams, Grenoble, Jérôme Millon, 1992, p. 186.

${ }^{28}$ Ibid., p. 188.

${ }^{29}$ M. Merleau-Ponty, «Le philosophe et son ombre», in CEuvres, op. cit., p. 1279: «L'homme peut faire l'alter ego que ne peut faire la "pensée", parce qu'il est hors-de-soi dans le monde et qu'une ek-stase est compossible avec d'autres ».
} 


\section{II - « L'autre existe » et le « je ne peux pas »}

C'est depuis la mise en question de ces deux figures du «je peux », qu'il s'agit désormais de faire s'esquisser le «je ne peux pas» constitutif de l'intercorporéité.

\section{$a-D u$ « je peux » au « je ne peux pas »: de l'impossible transposition}

C'est sur le corps comme «je peux » qu'est fondée la possibilité d'une aperception d'autrui par analogie. L'aperception analogique n'est pas un raisonnement, affirme Husserl, mais une "possibilité corporelle». Seulement, l'argument mobilisé par Scheler contre la théorie $\mathrm{du}$ "jugement par analogie » - et d'après lequel ce n'est jamais que moi que j'atteins par analogie ${ }^{30}$ - peut être développé sur le plan esthésiologique de la transposition spatiale imaginaire dans les vécus sensibles d'autrui. Admettre comme le fait Husserl, l'impossibilité de toute confirmation intuitive de cette transposition, n'est-ce pas admettre par là que, loin d'atteindre le vécu d'autrui, je n'atteins rien d'autre ainsi que mon propre vécu transposé en lui ? Lorsque Husserl affirme que j'appréhende l'autre " avec des phénomènes tels que je pourrais en avoir si j'allais là-bas (illic) et si j'y étais »31, il affirme en réalité que j'appréhende l'autre "avec des phénomènes tels que j'imagine que je pourrais en avoir si j'étais là-bas ». Et, plus donc que de reconnaître avec Husserl la dimension «imaginaire » d'une telle transposition, il s'agirait de souligner la dimension originaire et constitutive de l'impossibilité de sa réalisation effective. Si j'appréhende autrui comme "autre » $c^{\prime}$ est justement du fait de l'impossibilité a priori de réaliser intuitivement cette possibilité. Ainsi s'agit-il de maintenir fermement la distinction opérée par Husserl entre les deux types de "possibilités » que sont la "possibilité logique» ou possibilité formelle de se représenter et la «possibilité pratique » de « rendre intuitive cette possibilité $»^{32}$. L'exemple que prend alors Husserl est le suivant: si «je peux» me représenter que je bouge la table avec ma main sans médiation, comme je bouge ma main elle-même, «je ne peux pas » le faire - à savoir ici, je ne peux pas rendre intui-

\footnotetext{
${ }^{30}$ Voir M. Scheler, Wesen und Formen der Sympathie, M. S. Frings (éd.), Bern/Munich, Franckle Verlag, 1973, p. 234-235 ; trad. M. Lefebvre, Nature et formes de la sympathie, Paris, Payot \& Rivages, p. 327.

${ }^{31}$ Hua I, p. 146 ; MC, p. 191.

${ }^{32}$ Hua IV, p. 261-262 ; RPC, p. 354-355.
} 
tive cette possibilité33. Et n'en va-t-il pas ainsi de la transposition de $l^{\prime}$ « ici » (hic) au «là-bas » (illic) d'autrui, dont il est question dans la cinquième Méditation ? Si je peux me représenter ce que je percevrais si j'étais là-bas, à la place d'autrui, je ne peux pas le percevoir à proprement parler - à savoir, je ne peux pas «rendre intuitive cette possibilité ». Pour la simple raison que c'est aussi moi qu'autrui perçoit dès lors que je le perçois comme étant «là-bas ». C'est une impossibilité $a$ priori constitutive de la corporéité que de se percevoir soi-même comme étant «là-bas »- impossibilité plus prégnante encore que celle de percevoir autrui comme s'il était «ici». Pour être possible logiquement, il est a priori impossible de pénétrer effectivement le phénomène perceptif d'autrui, lorsque c'est justement moi qu'il perçoit de là-bas. $C^{\prime}$ est pourquoi l'on ne peut pas non plus objecter la possibilité pratique future de réaliser cette possibilité logique - la possibilité de modifier le « là-bas » (illic) en « ici » (hic), ou d'aller là où se trouve autrui. Lorsque je change de situation spatiale au moyen d'une « libre modification de mes sensations cinesthésiques » et que je me situe à la place d'autrui, les phénomènes perceptuels que j'ai $\mathrm{d}^{\prime}$ «ici » ne peuvent pas être les mêmes que ceux qu'avait autrui lorsque moi-même précisément, j'étais «là-bas». Plus que de percevoir les mêmes choses au moyen de "phénomènes différents » comme le concède Husserl ${ }^{34}$, ce ne sont justement pas les mêmes choses que je perçois.

L'on trouve déjà, chez Husserl, plusieurs figures de l'impossibilité corporelle. Plus que ne le fait Merleau-Ponty, Patočka reconnaît chez Husserl la dimension constitutive $\mathrm{du}$ « je ne peux pas »: «La conscience du "je peux" est une conscience essentiellement corporelle - le corps-sujet est ce qui, par essence, peut, faculté qui bien sûr, implique inversement que le corps peut aussi ne pas pouvoir $»{ }^{35}$.

Ce «ne pas pouvoir» peut s'entendre aux deux sens de $l^{\prime}$ «impossibilité » et de la «passivité». C'est une «impossibilité » tout d'abord au sens où Husserl lui-même reconnaît, aux côtés du «je peux » comme «liberté », l'importante part de facticité qu'implique la corporéité :

Tandis que, à l'opposé de toutes les autres choses, j'ai la liberté de changer à volonté ma position par rapport à elles [...], je n'ai pas en revanche la possibilité de m'éloigner de mon propre corps ou de l'éloigner de moi et, en

\footnotetext{
${ }^{33}$ La possibilité aux deux sens pratique et intuitive se recoupent ici - rendre intuitive cette possibilité logique c'est $l^{\prime}$ « accomplir ».

${ }^{34}$ Hua I, p. 146 ; MC, p. 190.

35 J. Patočka, Introduction à la phénoménologie de Husserl, op. cit., p. 187.
} 
conséquence, la diversité des apparences du corps propre est limitée d'une manière indéterminée ${ }^{36}$.

Cette « impossibilité » de n'être pas son propre corps interdit toute transposition intuitive de mon corps au corps d'autrui et annule la possibilité effective d'une transposition spatiale, sur laquelle repose la perception des vécus sensibles d'autrui. Rivé à mon corps et à mes phénomènes corporels, c'est l'impossibilité a priori de vivre ceux d'autrui qui constitue son altérité. Et de même que le "je peux » comprend la double possibilité pratique de l'intuition et de l'action, le «je ne peux pas» se distingue à son tour entre l'impossibilité de percevoir les vécus corporels d'autrui et le fait de «pâtir » par lui. Le « ne pas pouvoir» dont parle Patočka renvoie également à la passivité - au « pâtir » et au « subir », qui, au même titre que l' « agir » sont impliqués a priori par mon vécu corporel d'autrui. Husserl reconnaît également, à côté du «je peux» ou de "processus cinesthésiques libres », "des processus passifs auxquels la spontanéité n'a aucune part ». Il reconnaît, à côté du pouvoir d'agir ou du «je fais », un «ce qui m'est fait " ${ }^{37}$. Le mouvement du corps propre dans ce cas, « porte le caractère "psychique" du "subir" $\gg{ }^{38}$. Ce mode insigne de la corporéité n'est pas non plus sans jouer un rôle fondamental dans l'intercorporéité. Or, si de nombreux phénoménologues ont, comme Sartre, insisté sur le fait d'être vu ou perçu par autrui, peu auront insisté comme Levinas sur la possibilité de pâtir par lui - sur la « passivité » plus passive que toute passivité qui consiste en une inversion $\mathrm{du}$ 《je peux» dans le fait d'être «exposé » à autrui dans la vulnérabilité.

\section{b- De la compossibilité à l'impossibilité : de l'impossible réversibilité}

Le «je ne peux pas» constitutif de l'intercorporéité peut être également révélé depuis une mise en cause du principe de réversibilité sur lequel repose, chez Merleau-Ponty, la compossibilité. La « réversibilité » du sensible désigne cette propriété essentielle du corps propre d'être à la fois sentant et senti - la possibilité pour celui qui voit d'être vu et pour celui qui touche d'être touché. Ce que montre déjà l'exemple que prend Husserl au second tome des Ideen, de la possibilité pour le corps propre de se toucher lui-même et d'être

\footnotetext{
${ }^{36}$ Hua IV, p. 159 ; RPC, p. 224.

${ }^{37}$ Ibid., p. 159 ; p. 225.

${ }^{38}$ Idem.
} 
«perçu de l'extérieur ${ }^{39}$. Merleau-Ponty reprend cet exemple pour expliciter la perception du corps d'autrui. Déjà autre à lui-même, mon corps n'a pas de difficulté à percevoir un autre corps. Inhérente à ma propre corporéité, l'altérité en jeu dans l'intercorporéité sépare tout autant mon corps de lui-même, qu'elle ne sépare mon corps du corps d'autrui : "Ma main droite, écrit Merleau-Ponty, assistait à l'avènement du toucher actif dans ma main gauche. Ce n'est pas autrement que le corps d'autrui s'anime devant moi, quand je sers la main d'un autre homme ou quand seulement je la regarde ${ }^{40}$.

C'est cet argument de la possibilité d'une extension de la réversibilité de mon corps au corps d'autrui, qu'il convient ici d'interroger. Lorsque l'on sent son propre corps, comme l'écrit déjà Husserl : «nous avons la même sensation redoublée dans les deux parties du corps, parce que chacune est pour l'autre une chose extérieure qui touche et qui produit des effets et que chacune en même temps est corps propre ${ }^{41}$. C'est ce redoublement de sensations qui fait défaut justement, dans le cas d'un contact avec un corps étranger, aussi organique soitil lui aussi. Lorsque je touche ma main droite avec ma main gauche, je me sens et je me sens être senti - mes deux mains étant à la fois sentantes et senties. Lorsque je serre la main d'autrui, je me sens certes être senti, mais je ne le sens pas lui me sentir ni me sentir senti par lui - au sens du moins où je n'en ai pas de sensations. Alors que dans la perception du corps propre je sens un corps qui me sent en retour et que je sens me sentir - au sens où j'en ai la sensation redoublée; dans la perception d'un corps organique étranger, je sens un corps qui me sent en retour mais que je ne sens pas me sentir - au sens où je n'en ai pas de sensations. Si ma main droite est à la fois sentante et sentie, l'autre main, celle d'autrui, n'est que sentie. Il n'y a donc pas de complète réversibilité de mon corps à celui d'autrui. L'impossibilité a priori d'avoir des sensations internes du corps d'autrui, aussi évident cela puisse-t-il paraître, est, plus que ne l'est la possibilité de sa sensation externe, constitutive de l'intercorporéité. Et si je peux a contrario me sentir moi-même de l'extérieur - avoir une sensation externe de mon propre corps - l'extériorité dont il jouit alors, ne peut être comparée à l'extériorité du corps d'autrui. Comme l'écrit pourtant Merleau-Ponty: «Entre ma conscience et mon corps tel que je le vis, entre ce corps phénoménal et celui d'autrui tel que je le vois du dehors, il existe une relation interne qui fait apparaître

\footnotetext{
${ }^{39}$ Ibid., p. $144-145$; p. 207.

${ }^{40}$ M. Merleau-Ponty, « Le philosophe et son ombre », in CEuvres, op. cit., p. 1276.

${ }^{41}$ Hua IV, p. 145 ; RPC, p. 207.
} 
autrui comme l'achèvement du système ${ }^{42}$. L'achèvement d'un système de compossibilité dont l'existence d'autrui fait toujours déjà partie. Mais cette objection contre la possibilité d'étendre la réversibilité du sensible à la perception d'autrui, révèle une dimension $\mathrm{d}^{\prime}$ " extériorité » incompressible à même ce "système ». L'extériorité en ce sens radical de ce qui ne peut pas être intérieurement "senti ", n'est pas le seul produit théorique d'une conscience objectivante qui tend à s'enfermer en sa propre pensée en oubliant l'unité première du monde qui la contient ; ce n'est pas, comme le pense Merleau-Ponty, le fait d'une conscience adulte qui vient troubler la co-existence pacifique de l'enfance. Il est une extériorité absolue à même l'intercorporéité - et ce, depuis la prime enfance. La chair du sensible de laquelle nous participons corporellement, doit donc être repensée comme impliquant une "altérité » d'un tout autre type que celle qui sépare mon corps de lui-même. Une altérité qui n'est peut-être pas "incompossible», mais que la seule compossibilité ne suffit pas à expliciter. Pour suffire à expliciter la possibilité de l'empathie, le concept de «compossibilité » ne suffit pas à expliciter la possibilité, toute aussi signifiante, de l'absence d'empathie. Possibilité d'une absence d'empathie qui s'ancre justement dans l'impossibilité a priori d'une complète réversibilité. Toujours déjà avec autrui, organe d'une seule et même chair, je ne pourrais pas par exemple le craindre, être menacé par lui. Pour appartenir d'emblée à un même univers de compossibilité, autrui n'en reste pas moins, à de nombreux égards, « impossible » pour moi.

Plus encore que la voie égologique husserlienne qu'elle entend dépasser, cette conception de l'intercorporéité comme système de compossibilité échoue à rendre compte du rôle signifiant du «je ne peux pas » qui subsiste à même l'intercorporéité, et qui s'origine à même la chair, dans l'impossibilité d'une complète réversibilité. C'est parce qu'il n'est pas de parfaite réversibilité de mon corps à celui d'autrui, que ce dernier, irréductible à tout système de compossibilité, conserve de l'imprévisibilité.

\section{c-Levinas : du corps-maître au corps-esclave}

Telle qu'elle fut pensée par Levinas, la relation à autrui excède tout système de compossibilité. Contre l'affirmation de Merleau-Ponty d'après laquelle autrui n'est jamais perçu de face, autrui n'est plus

\footnotetext{
${ }^{42}$ M. Merleau-Ponty, Phénoménologie de la perception, in CEuvres, Paris, Gallimard, 2010, p. 1053.
} 
seulement chez Levinas celui «avec » lequel j'agis, mais celui sur lequel je peux agir et qui, en retour, peut agir sur moi. D'où la possibilité dans Totalité et Infini de vouloir tuer autrui, et celle inverse dans Autrement qu'être, d'être exposé à lui. Ce qui d'autrui ne revient pas au même - ou ne revient pas à moi - peut être tour à tour menacé et menaçant. C'est l'imprévisibilité d'autrui - et l'impossibilité donc de toute réversibilité - qui le rend à la fois infiniment résistant à mes pouvoirs, et infiniment menaçant. Cette imprévisibilité réactive dans Totalité et Infini - face à mon pouvoir de tuer, autrui peut $\mathrm{m}^{\prime}$ opposer "l'imprévisibilité même de sa réaction " ${ }^{43}$ - devient "offensive " dans Autrement qu'être - c'est l'imprévisibilité d'une venue à laquelle je ne peux pas me préparer, et qui me constitue dans ma vulnérabilité. Et c'est dans ce dernier cas seulement que l'imprévisibilité d'autrui est pensée en sa dimension charnelle, comme le fait d'une intercorporéité. Si Levinas parle bien de la résistance d'autrui à mes "pouvoirs » dans Totalité et Infini, c'est moins par son corps qu'autrui résiste, que par ce qui en lui justement s'y soustrait. Le visage qui résiste ainsi, n'est pas du monde et signe déjà vers l'au-delà de toute corporéité. $L^{\prime}$ « impossibilité éthique » qui structure la relation sociale, ne se joue pas encore à même la chair ${ }^{44}$. D'où " l'impossibilité de tuer » autrui à proprement parler ${ }^{45}$. C'est dans la troisième section $\mathrm{d}^{\prime}$ Autrement qu'être, que l'expérience insigne qu'est celle d'autrui est repensée en termes de corporéité ; et que la corporéité doit être à son tour repensée au-delà de toute jouissance: "La signification - l'un pour l'autre -, écrit Levinas, n'a de sens qu'entre êtres de chair et de sang ${ }^{46}$. Et, de même que la chair du social excède la compossibilité, le corps propre est moins vécu ici sur le mode $\mathrm{du}$ " je peux » que sur celui de «l'exposition à la blessure » qu'est la vulnérabilité. Comme l'écrivait déjà Sartre :

Ce que je saisis immédiatement lorsque j'entends craquer les branches derrière moi, ce n'est pas qu'il y a quelqu'un, c'est que je suis vulnérable, que j'ai un corps qui peut être blessé, que j'occupe une place et que je ne puis, en aucun cas, m'évader de l'espace où je suis sans défense ${ }^{47}$.

\footnotetext{
${ }^{43}$ E. Levinas, Totalité et Infini, Essai sur l'extériorité, La Haye, Martinus Nijhoff, Kluwer Academic, 1971, p. 215.

${ }^{44} \mathrm{Ibid} .$, p. 185.

${ }^{45}$ Ibid., p. 216.

${ }^{46}$ E. Levinas, Autrement qu'être ou au-delà de l'essence, La Haye, Martinus Nijhoff, Kluwer Academic, 1974, p. 119.

${ }^{47}$ J.-P. Sartre, L'être et le néant, Paris, Gallimard, 1943, p. 316.
} 
Et, si Levinas ne parle pas explicitement de la corporéité comme d'une inversion $d u$ « je peux », n'en parle-t-il pas néanmoins comme d'une inversion $\mathrm{du}$ " conatus de l'esse » ? Rencontrer le corps d'autrui, c'est non seulement cesser de pouvoir persévérer, mais c'est être soimême «exposé ». D’où la passivité du corps, "plus passive que toute passivité ». Le corps propre, dans l'intercorporéité, doit être repensé comme "corps-effet » ${ }^{48}$, depuis la possibilité persistante du "pâtir » ou du «subir», dans cette «exposition à la blessure» par l'autre qu'est la «vulnérabilité ». «La vie atteste, dans sa peur profonde, cette inversion toujours possible du corps-maître en corps-esclave $»^{49}$. Inversion constitutive d'une intercorporéité qui ne désigne plus, ni la relation externe de deux corps étrangers, ni la relation interne de deux organes d'une même chair, mais bien la déchirure diachronique qui travaille à même cette dernière relation - la possibilité d'une absolue séparation à même l'intercorporéité. Moins donc le système de nos compossibilités, que l'irréductible diachronie qui travaille à le dénouer et qui fait que le corps de l'autre peut être craint ou désiré. Voire même que mon corps propre peut être tout entier investi par l'autre - devenant à son tour autre à lui-même - comme c'est le cas chez Levinas, $\mathrm{du}$ « corps maternel ».

Husserl puis Merleau-Ponty reposent la question traditionnelle de l'intersubjectivité en termes d'intercorporéité - comme relation charnelle au corps d'autrui. Et c'est très succinctement que nous avons tâché d'interroger le mode de la corporéité qui est impliqué par sa dimension essentiellement relationnelle - la thèse selon laquelle, dans sa relation au corps d'autrui, le corps se tiendrait d'abord ou seulement, sur le mode $\mathrm{du}$ «je peux». Contre la possibilité d'une transposition effective, et contre la possibilité d'une parfaite réversibilité, il s'agissait de faire apparaître le rôle constitutif de l'impossibilité dans l'intercorporéité. Impossibilité qui tenait chaque fois à l'impénétrabilité des vécus corporels d'autrui, et qui, loin de mettre en cause la possibilité de sa perception, la constitue bien plutôt. L'intercorporéité serait moins fondée en cela sur la possibilité de l'empathie que sur son irréductible résidu d'impossibilité. Impossibilité au-delà de tout " système de compossibilité » dans laquelle s'ancre la possibilité d'un agir non plus « avec » mais «sur » autrui - et la possibilité corrélative d'un "pâtir » par lui. Dans un monde de stricte compossibilité où tous mes pouvoirs corporels impliqueraient d'ores et déjà l'existence

\footnotetext{
${ }^{48}$ Voir à ce propos E. Levinas, Totalité et Infini, op. cit., p. 117.

${ }^{49} \mathrm{Idem}$.
} 
d'autrui, il n'y aurait pas de vulnérabilité. D’où la nécessité lévinassienne de faire retour de la suffisance du « corps propre » à la passivité et à l'impuissance du " corps-effet».

Or, comme l'écrivait déjà Patočka : "le "je peux" du corps propre est par ailleurs le corrélat d'un "je dois" et d'un "je ne peux pas" ${ }^{50}$. $C^{\prime}$ 'est au détour de cette citation que l'on comprend finalement pourquoi la manifestation de l'impossibilité à même la chair intercorporelle, répond à la nécessité éthique d'une pensée de l'intercorporéité - pourquoi le «je ne peux pas » est déjà un «je dois ». La rencontre d'autrui n'est pas chez Levinas, le simple fait d'un rapport de force dès lors que l'autre est aussi un corps, et qu'il est lui aussi infiniment nu et exposé. Loin de m'opposer sa toute-puissante corporéité - comme dans l'équivalent charnel d'une lutte à la mort - autrui m'oppose sa propre vulnérabilité. Et c'est ainsi seulement que le «je ne peux pas » peut signifier un « je dois ». Violemment exposée à la vulnérabilité de la chair de l'autre, ma chair se donne, au lieu de s'opposer. Seules de telles descriptions du corps propre permettraient d'interroger la manière dont l'éthique peut être vue se déployer à même la chair de l'intercorporéité.

${ }^{50}$ J. Patočka, Introduction à la phénoménologie de Husserl, op. cit., p. 189. 